\title{
Oleuropein modulates over-proliferation of keratinocytes in mice with imiquimodmediated psoriasis and inhibits differentiation of Th17 cells through the JAK3/STAT3 axis
}

Quan Shi1,2, Qi He ${ }^{1,2}$, Weiming Chen ${ }^{1,2}$, Jianwen Long ${ }^{1,2}$, Bo Zhang ${ }^{1,2}$

\author{
${ }^{1}$ Department of Dermatology, Hubei Provincial Hospital of Traditional Chinese \\ Medicine, Wuhan, Hubei, China \\ ${ }^{2}$ Department of Dermatology, Hubei Province Academy of Traditional Chinese \\ Medicine, Wuhan, Hubei, China
}

Submitted: 7 August 2020

Accepted: 11 September 2020

Arch Med Sci

DOI: https://doi.org/10.5114/aoms.2020.100642

Copyright (c) 2020 Termedia \& Banach

\section{Abstract}

Introduction: Oleuropein (OLP) is polyphenol obtained from olive oil; it is proved in Chinese traditional medicine for its use in disorders including autoimmune and inflammatory disorders. Psoriasis (PSR) is an autoimmune and inflammatory disorder triggered by T-helper-17 (Th17) cells.

Material and methods: We developed an imiquimod (IMQ)-mediated PSR model in mice to study the anti-inflammatory role of OLP in psoriasis. The mice were given $50 \mathrm{mg} / \mathrm{kg}$ and $100 \mathrm{mg} / \mathrm{kg}$ dose of OLP. Histology was done to assess the inflammation of lesions. Western blot analysis was done for JAK3/STAT3 in isolated T cells, expression of ROR $\gamma$ t was done by RT-PCR. The In silico molecular docking studies were done for interaction of OLP with target protein STAT3 and JAK3.

Results: Treatment of OLP attenuated proliferation in IMQ-mediated keratinocytes, improved infiltration of CD3+ cells in the skin lesions and in CD4+ and CD8+ T cells and also ameliorated the levels of cytokines. In in vitro studies in isolated T cells, OLP blocked the differentiation of Th17 cells and also the levels of IL-17 and the JAK3/STAT3 pathway. The in silico docking showed that OLP had potential binding affinity with JAK3 and STAT3 which was parallel to in vivo and in vitro findings.

Conclusions: OLP ameliorates psoriasis skin lesions by blocking Th17-mediated inflammation. OLP may be an interesting molecule for treating autoimmunity in psoriasis.

Key words: oleuropein, psoriasis, imiquimod, Th17, JAK3/STAT3.

\section{Introduction}

Psoriasis (PSR) is an inflammatory autoimmune disorder of skin characterized by aberrant differentiation and excessive proliferation of skin cells accompanied with infiltration of immune cells. Events such as proliferation of keratinocytes and infiltration of immune cells cause psoriatic lesions leading to erythematous and red scales on skin along with itching and pain [1]. It has been evidenced that upon exposure of genetically susceptible individuals to various environmental factors such as physical trauma and infection by microorganisms, the inflammatory pathway in psoriasis

\author{
Corresponding author: \\ Dr. Qi He \\ Department \\ of Dermatology \\ Hubei Provincial Hospital \\ of Traditional Chinese \\ Medicine \\ Hubei Province Academy \\ of Traditional Chinese \\ Medicine \\ 430074 Wuhan \\ Hubei, China \\ Phone/fax: +86 027 \\ 88929294 \\ E-mail: Catty0004@163.com
}


plaques is induced via dendritic cells; these cells are responsible for producing interleukins which are responsible activating the $T$ helper $(T h)$ cells $[2,3]$. Although the driving mechanisms behind development and progression of PSR are still not well understood, many studies have evidenced involvement of Th17 cells in promoting PSR lesions [4]. The Th17 cells are found in lesions resulting in an increased number of Th17 cells in the circulation around the lesions and are directly related to the affected PSR area and severity index (PASI) [5]. The Th17 cells are generated by differentiation of CD4+ cells under the influence of interlukin-6 (IL-6), IL-1 and transforming growth factor $\beta$ (TGF- $\beta$ ) whereas the survival is governed by IL-23. The IL-17 family cytokines originate mainly from Th17 cells; also IL-17 is identified to be the main cytokine responsible for PSR [6]. IL-17A primarily targets receptors present on the surface of dendritic cells, endothelial cells, keratinocytes and dermal fibroblasts [7]. The main clinical feature of PSR is scaling papules or lesions of size less than $1 \mathrm{~cm}$ and plaques; also PSR may lead to development of trauma at the site of injury, which is called Koebner's phenomenon. PSR if not managed properly can lead to erythroderma. In addition, PSR may occasionally involve the oral mucosa or tongue [8]. Management of PSR involves use of synthetic molecules such as brodalumab, ixekizumab and secukinumab, which are IL17A inhibitors which have shown promising results in clinical trials (phase-II) and have been utilized in treating PSR [9]. However, use of synthetic derivatives has its own disadvantages; hence searching for novel bioactive derivatives could be a safe and better approach.

OLP is found to be the glycated derivative of the major secoiridoid precursor 3,4-dihydroxyphenylethanol-elenolic acid (3,4-DHPEA-EA) obtained from olive oil and is responsible for the bitter taste in its leaves. Olive oil has been found to be effective in treating topical disorders such as atopic dermatitis or psoriasis [10]. OLP has been demonstrated to possess multiple activities such as anti-inflammatory [11], immune-mediated anti-inflammatory disorders [12], diabetes [13] and cancer [14]. Nevertheless, the specific molecular mechanism of OLP associated with inflammation remained unclear. Previous reports have shown that OLP suppresses neutrophil, macrophage, eosinophil and Th17 responses $[15,16]$. In addition, OLP has been reported to inhibit NF- $\kappa B$ and phosphorylation of p38 [17]. In the current study the role of OLP in PSR was investigated in both in vivo and in vitro models; we also studied the mechanism involved.

\section{Material and methods}

\section{Animals and treatment}

The mouse model of PSR was created using $\mathrm{BALB} / \mathrm{c}$ mice (male) weighing approximately $20 \mathrm{~g}$ and aged 7 to 8 weeks. The mice were bought from the Animal Center of Hubei Provincial Hospital of Traditional Chinese Medicine, China and were housed under controlled conditions with water ad libitum and pellet diet. The animal studies followed the guidelines provided by the ethical board of Hubei Provincial Hospital of Traditional Chinese Medicine, China. The animal experiments were approved by the ethical review board of Hubei Provincial Hospital of Traditional Chinese Medicine, China. The animals were randomly divided into 5 groups $(n=8)$. The hairs on the back of mice were removed; among them the four groups received topical treatment of IMQ (5\%) daily to establish the IMQ-induced mouse model of PSR [18]. The mice of the control group did not receive treatment with IMQ. Oleuropein (OLP) (Sigma Aldrich USA) was used without any further purification by solubilizing in DMSO followed by dilution in isotonic solution of sodium chloride (0.9\%) to achieve a concentration $<0.1 \%$ and administered orally. The negative control group IMQ mice received vehicle (DMSO and isotonic saline) treatment, the positive control IMQ mice received methotrexate (MTX) $1 \mathrm{mg} / \mathrm{kg}$ as standard (choice of drug in PSR), and the OLP was given at two concentrations: $100 \mathrm{mg} / \mathrm{kg}$ in IMQ mice was high dose, $50 \mathrm{mg} / \mathrm{kg}$ in IMQ mice was low dose [19]. All the defined treatment protocols started in the mice exposed to IMQ once in a day for 1 week. During the treatment course severity features were studied and recorded with the help of the modified PASI scoring system [20]. The scores represented the inflammation severity. After treatments for 7 days, the experimental mice were submitted to cervical dislocation and sacrificed after submitting them to pentobarbital anesthesia followed by collection of serum and skin samples.

\section{Isolating mouse CD4+ T cells and differentiation of Th17 in vitro}

The isolated splenic $T$ cells were submitted to negative selection for purification using the cell isolation kit as per the supplied instructions. The purity of CD4+ cells was evaluated by FACS sorting using the anti-CD4 FITC. FSC values were used for selecting the viable cells; after this the Th17 cells were separated and represented $>98 \%$ values of the total cells. The cells were transferred to 12-well plates and subjected to seeding having anti-CD3 and soluble anti-CD28 antibodies with $2 \times 10^{6}$ cells/well in RPMI media (Thermo Fisher USA) loaded with inactivated fetal bovine serum (15\%). For differentiation of Th17 cells, the splenic $T$ cells were incubated along with TGF- $\beta$ ( $5 \mathrm{ng} / \mathrm{ml})$, IL-1 $\beta$ (10 ng/ml), IL-6 (10 ng/ml), IL-23 (10 $\mathrm{ng} / \mathrm{ml}), \mathrm{IFN}-\gamma-\mathrm{IL}-2(5 \mu \mathrm{g} / \mathrm{ml})$ and IFN- $\gamma$-IL-4 antibodies as described in earlier studies [21]. The differentiation of Th17 was analyzed by flow cytometer as reported earlier [22]. OLP used for treatment was solubilized in DMSO and exposed to cells at vari- 
ous concentrations for $72 \mathrm{~h}$. The control group cells were treated with an equivalent amount of DMSO as used in other group cells.

\section{Cell counting Kit-8 assay}

The cell viability studies were performed using Cell counting Kit-8 (CCK-8) assay (Sigma Aldrich USA) following the supplied instructions 3 days after induction of Th17 differentiation. The optical density (OD) was recorded for at least 3 wells/group for calculating cell proliferation.

\section{Flow cytometry study}

Staining with CD4-FITC, CD8-PE and CD3-APC antibodies was performed on cells. Prior to IL-17A staining, the cells were maintained with BG Golgi inhibitor of protein transporter and Leukocyte Activation cocktail (Fisher Scientific, USA) for $6 \mathrm{~h}$ and then suspended in permeability solution followed by anti-IL-17A PE staining. The blood cytokine quantification was done with BD CBA Human Th1/Th2/ Th17 Cytokine Kit following the supplied instructions (Fisher Scientific, USA).

\section{Immunofluorescence}

and immunohistochemical staining

For preparing the sections, the skin layer from the back part of mice bearing lesions was harvested and stored in formalin (10\%) and embedded in paraffin wax. The skin containing wax blocks were submitted to hematoxylin and eosin staining (H\&E), antirabbit CD3 PCNA (1: 500) and IL-17 (1:500) antibodies. The analysis of staining was evaluated with the help of fluorescence microscopy (Olympus, Japan).

\section{Western blot analysis}

Expression of proteins was done by western blot analysis, for that the skin samples were submitted to lysis and the proteins were separated in SDSPAGE (10\%) membranes. The membranes were incubated along with anti-SCOSE3 (1: 1000), JAK2 (1: 1000), JAK3 (1: 1000) and p-STAT3 (1: 1000) antibodies, and anti-actin rabbit antibodies followed by densitometric analysis.

\section{Real-time polymerase chain reaction (RT-PCR)}

For RT-PCR analysis, the skin lesion was processed to extract total RNA with the help of TRIzol reagent (Invitrogen USA) followed by purification with an RNA clean-up kit. The cDNA was produced with the help of a cDNA synthesis kit (Sigma Aldrich USA) using primers; the sequences of primers were RORr (forward and reverse) AACTGGCTTTCCATCATCATCTCTG, GGGAAGGCGGCTTGGACCACGA, actin (forward and reverse) GCCTTCCTTCTTGGGTAT and GGCATAGAGGTCTTTACGG. The gene expression was analyzed using the TaqMan Real-time PCR system, with actin used as loading standard.

\section{Ramachandran plot}

The Ramachandran plot (homology modeling) was performed for IL-17, JAK2 and STAT3; the sequences of the three proteins were downloaded from the NCBI database. SWISS modeling was performed with the help of the SWISS-MODEL server for developing the homology model of IL-17, JAK2 and STAT3. The software employed for the study included Autodock Vina tools 1.5.6 (The Scripps Research Institute), PyMol molecular graphics system version 2.4.0 (Schrodinger LLC), ACD/ChemSketch (ACD/ Labs). The protein sequences of IL-17, JAK and STAT3 were taken from the universal protein source. The web server of SWISS-MODEL was used for building the homology model. BLAST search was performed for finding the target sequences against the primary amino acid sequence. The best quality templates were selected for building the homology model.

\section{Ligand, protein preparation and molecular docking}

The structure of the target ligand, i.e. OLP, was downloaded from the PubChem database server as an SDF file. The ligand was prepared for docking with the help of Autodock vina MGL tools by adding hydrogens and modulating the charges adopting the Kollman approach and finally the PDB file was obtained. The homology modeling generated proteins were also processed by removing any water molecules and modifying the charges similarly as done for the ligand. The molecular docking simulation of the ligand OLP was done by Autodock Kollman and Gasteiger charges in both the ligand as well as the protein. The grid map was created with the help of Autogrid 4. The grid box was prepared and selection of the area on the protein structure to be mapped was defined by providing the coordinates. The grid box dimensions ( $x, y$ and $z$ coordinates) for IL17 were 22.5, 22.5 and 22.5, for JAK2 the dimensions were 33.5, 33.5 and 33.5, whereas for STAT3 the coordinates were 25.5, 25.5 and 25.5. The Lamarckian genetic algorithm was used for minimization and optimization of energies in the docking simulation process.

\section{Ethics approval and consent to participate}

The experiments were approved by the institutional animal ethical committee of Hubei Provincial Hospital of Traditional Chinese Medicine, Hubei Province Academy of Traditional Chinese Medicine China.

\section{Statistical analysis}

The results are depicted as mean \pm standard deviation (SD). For comparing groups, Newman- 
Keuls post hoc test and one-way analysis of variance (ANOVA) were used. Unpaired Student's $t$-test was used for comparing 2 groups, and a value of $p<0.05$ was defined as statistically significant.

\section{Results}

Treatment with oleuropein ameliorates IMQ-induced differentiation and proliferation of keratinocytes in psoriatic mice

To study whether OLP exerted a beneficial effect in the condition of PSR, the experimental PSR mice were treated with OLP. The mouse model of
PSR was created by IMQ; after 7 days the skin of mice was seen with erythema, thickening of skin and scaling due to IMQ-induced skin lesions. It was observed that both the selected doses of OLP (50 and $100 \mathrm{mg} / \mathrm{kg}$ ) produced no alterations in body weight compared to control mice. It was also observed that OLP-treated mice resulted in smooth skin texture, shallower erythema and less dense scales compared to control mice (Figures 1, A, B).

The IMQ-induced lesions on the skin showed increased ridges and epidermal hyperplasia, the ridges were more elongated with presence of inflammatory cells in the epidermal layer of skin, but
A

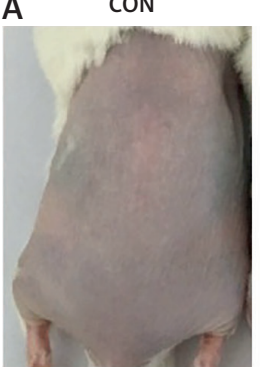

B

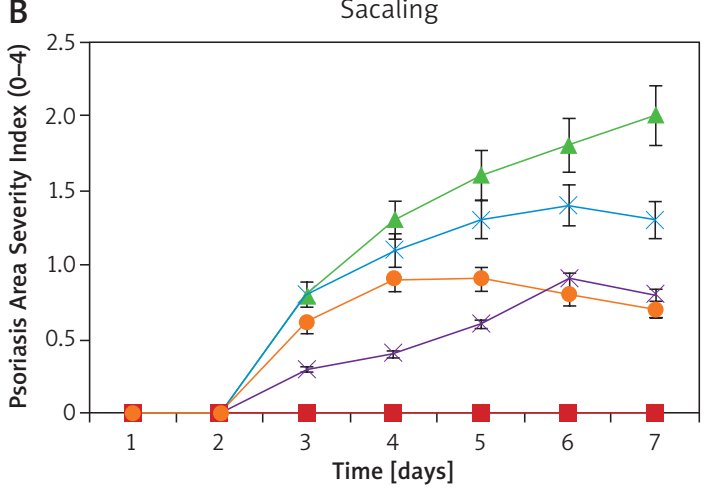

$\because \mathrm{CON} \triangle \mathrm{IMX} \nsucc \mathrm{MTX} \nsucc \mathrm{OLP}-50 \longrightarrow$ OLP-100

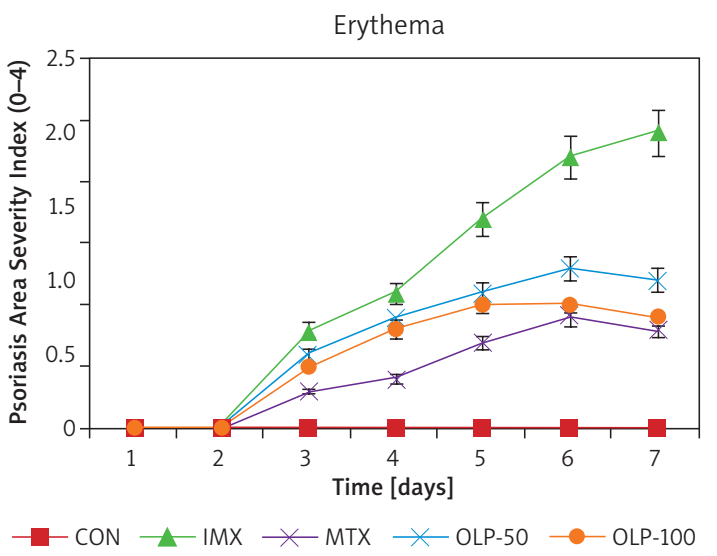

MTX
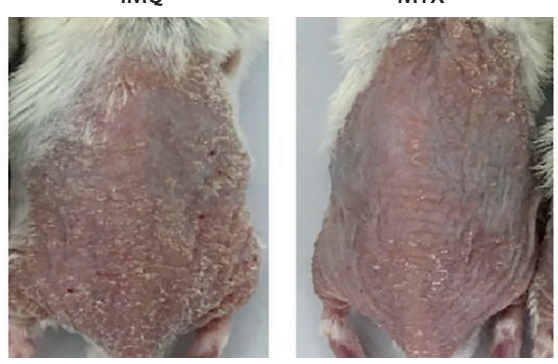

OLP-100

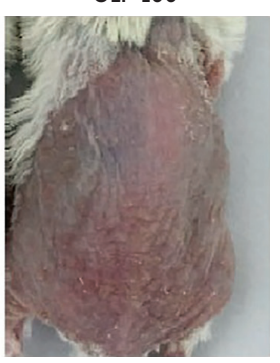

Thickness

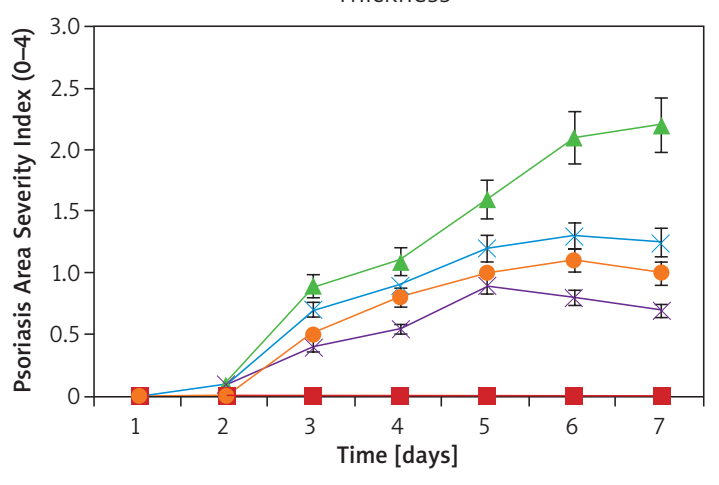

- CON $\triangle \mathrm{IMX} \nsucc \mathrm{MTX} \longrightarrow \mathrm{OLP}-50 \longrightarrow \mathrm{OLP}-100$

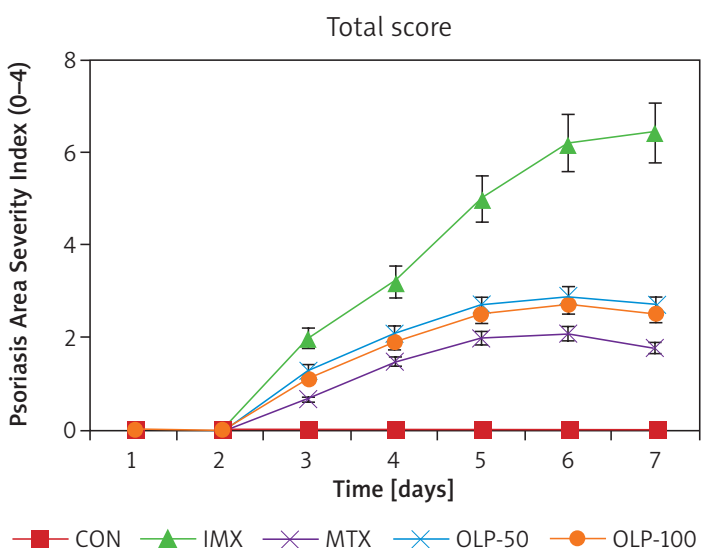

Figure 1. Oleuropein treatment attenuated the IMQ-mediated lesions of skin. A - The mice were submitted to topical application of IMQ, the IMQ mice were found to have psoriasis lesions on skin surface but not in control mice. The mice treated with methotrexate (MTX) or OLP-50 and $100 \mathrm{mg} / \mathrm{kg}$ dose showed improvement in lesions. B - Results (mean \pm SD) of Psoriasis Area Severity Index for scaling, erythema, thickness and total score ${ }^{*} P<0.05$ compared to IMQ-induced mice. 
C

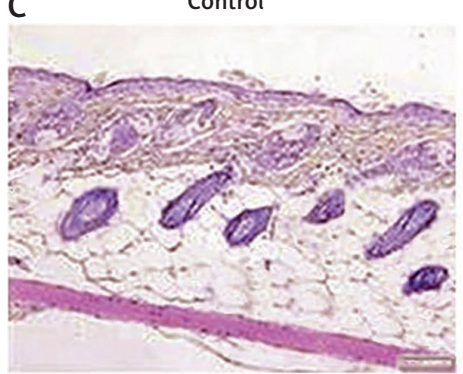

OLP-50

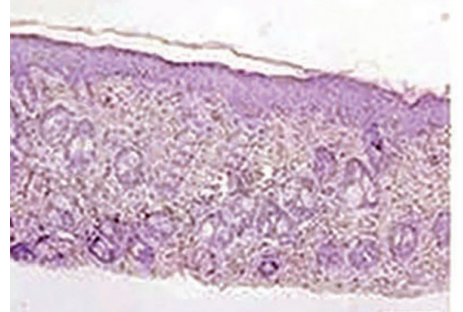

$\longrightarrow$
IMQ

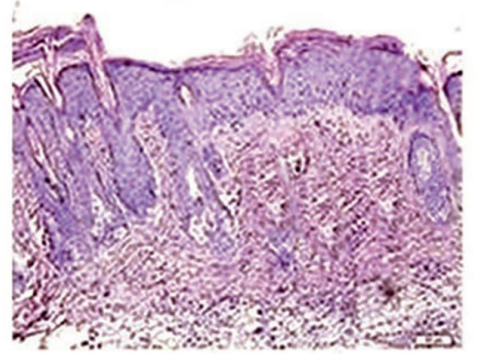

OLP-100

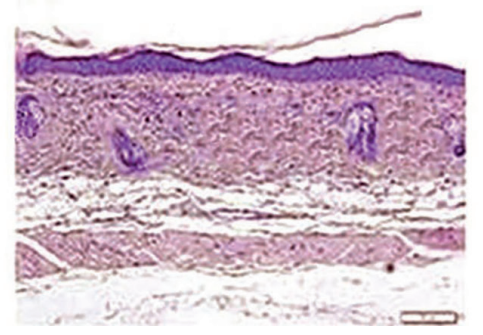

MTX

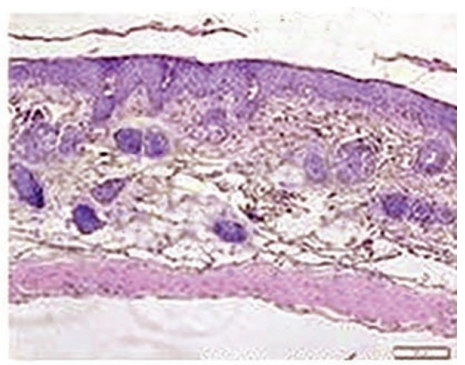

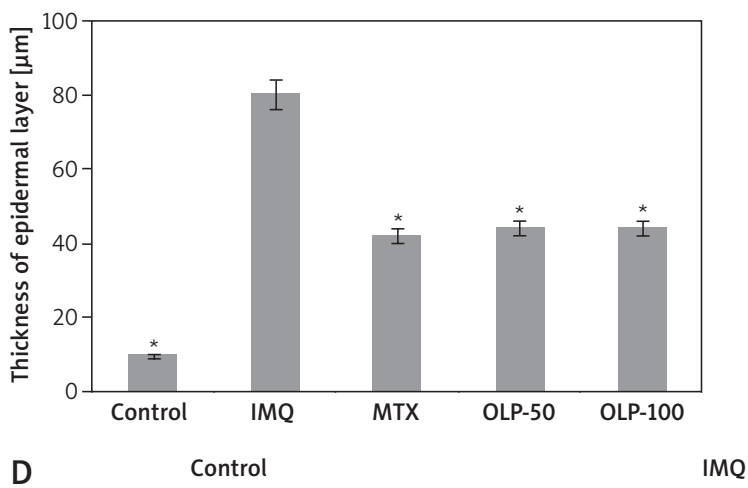

MTX

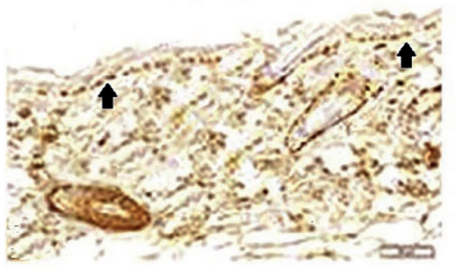

OLP-50
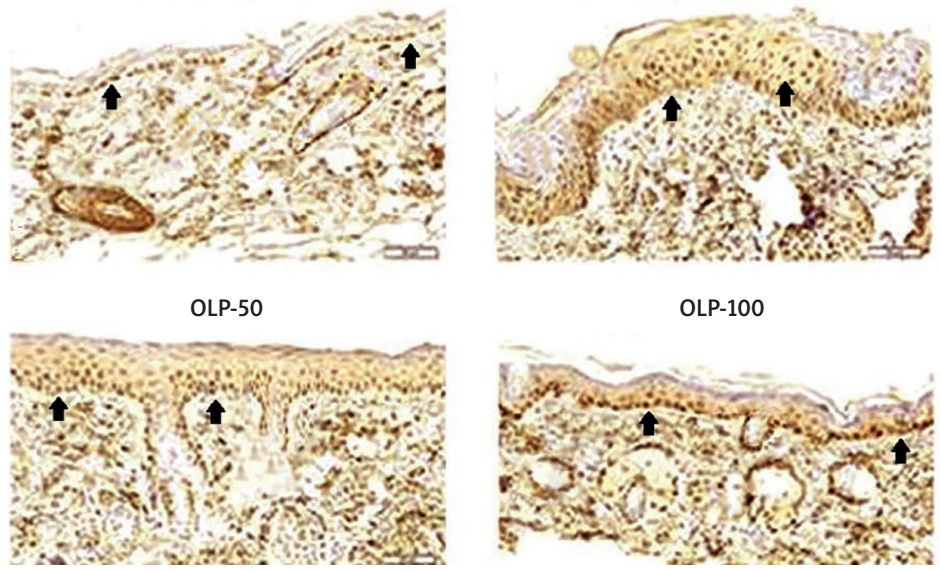

OLP-100
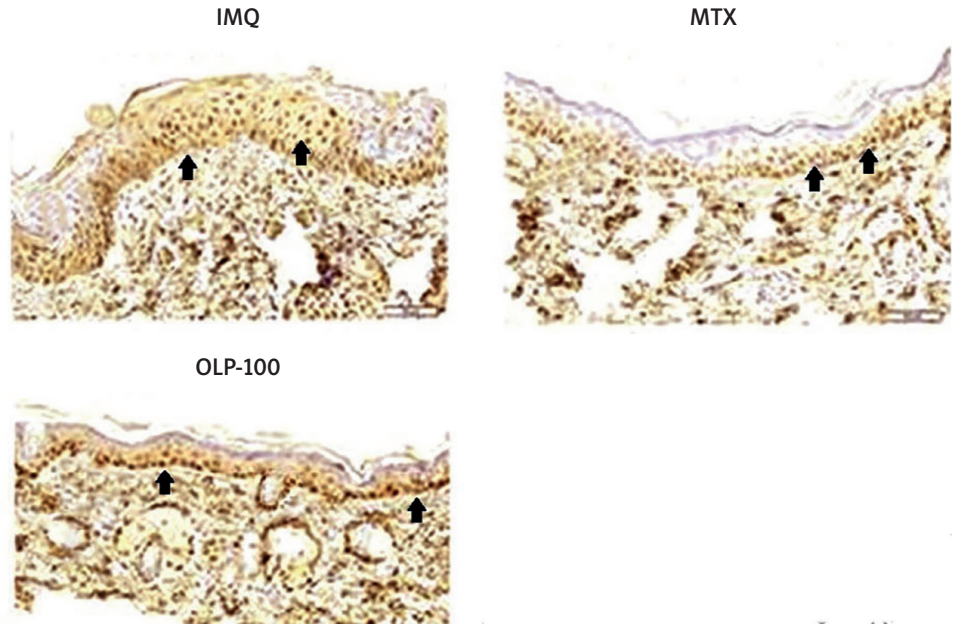

Figure 1. Cont. C - Hematoxylin and eosin staining of skin harvested from back of mice and thickness of epidermal layer. D - Immunohistochemical staining for proliferating cell nuclear antigen (PCNA) also showing positive cells in epidermal layer ${ }^{\star} P<0.05$ compared to IMQ-induced mice. 
the treatment of OLP decreased the thickness of the epidermal layer significantly and also ameliorated the IMQ-mediated PSR (Figure $1 \mathrm{C}$ ). It was observed that the levels of PCNA were decreased in skin lesions of mice treated with OLP, indicating that OLP decreased the IMQ-induced proliferation of keratinocytes (Figure $1 \mathrm{D}$ ). The outcomes clearly indicated that the treatment of OLP ameliorated IMQ-induced differentiation of keratinocytes.

\section{Treatment with oleuropein blocked the T cell-induced infiltration of inflammatory cells in psoriatic mice}

We studied the effect of OLP on infiltration of inflammatory cells. It was observed that CD3+ cells were infiltered in skin lesions of PSR mice but were decreased on treatment with OLP (Figure 2 A). It was found that the spleen and lymph nodes in PSR mice were enlarged, and the number of both $T$ cells, i.e. CD4+ and CD8+, was also increased in both of the glands. Treatment with OLP decreased the weight of both spleen and the lymph nodes and both the tissues showed a decrease in number of CD4+ and CD8+ cells in IMQ mice (Figure 2 B). Treatment with IMQ resulted in over-expression of blood levels of cytokines, suggesting activation of systemic immunity. The blood levels of IL-17A, IL-10, IL-4, IL-2, IL-6, IFN- $\gamma$ and TNF- $\alpha$ were increased significantly in IMQ-induced mice. Treatment with OLP significantly decreased the blood levels of IL-2, IL-4, IL-6, IL-17A, TNF- $\alpha$ and IFN- $\gamma$ along with IL-10 (Figure 2 C).

\section{Treatment with oleuropein blocked differentiation of Th17 cells and IL-17A release in psoriasis mice}

To study how OLP affects the functioning of Th17 cells, CCK-8 assay was performed to investigate whether OLP affected viability of Th17 cells. At very low (5 to $20 \mu \mathrm{g} / \mathrm{ml}$ ) concentrations OLP failed to alter the viability of Th17 cells, but at the concentration of $100 \mu \mathrm{g} / \mathrm{ml}$ it suppressed the viability of Th17 cells (Figure 3 A). To study the effect of OLP on differentiation of Th17 cells, flow cytometric analysis and bead based immunoassay were done to study the IL-17A+ and IL-17A respectively prior
A

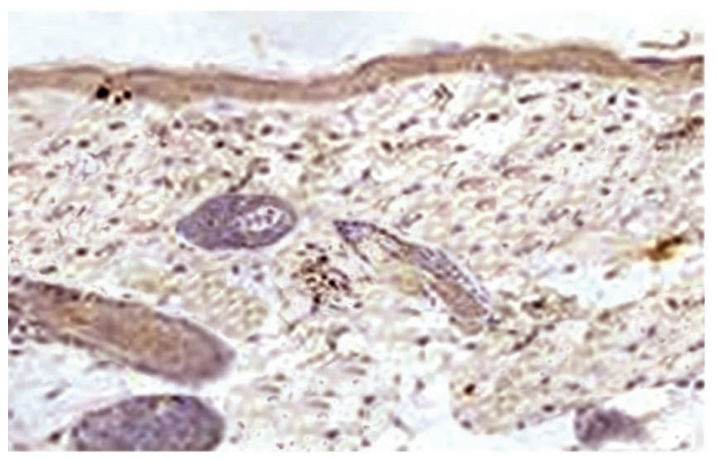

MTX
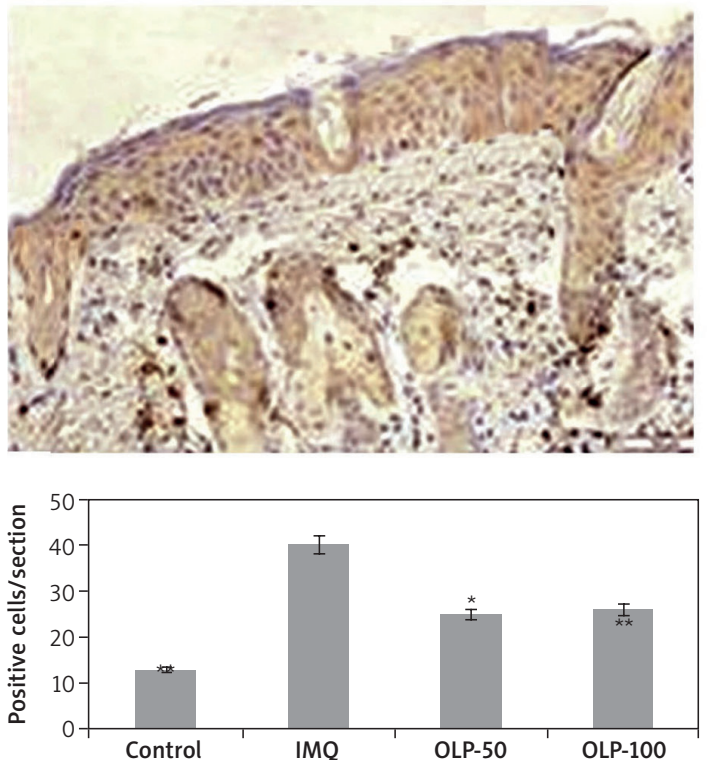

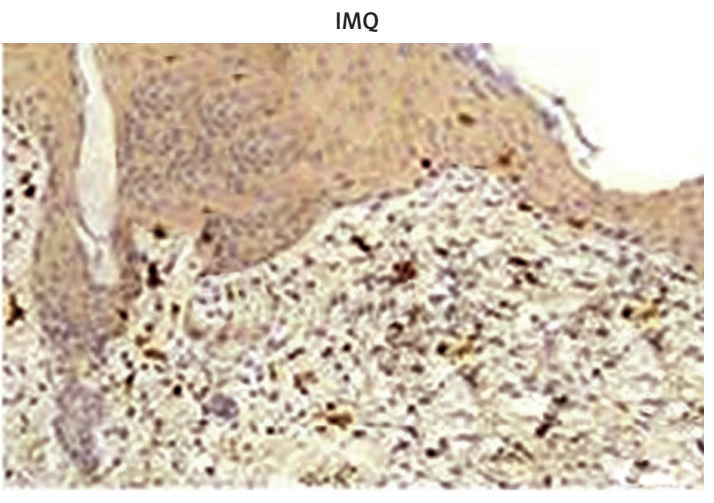

OLP-100

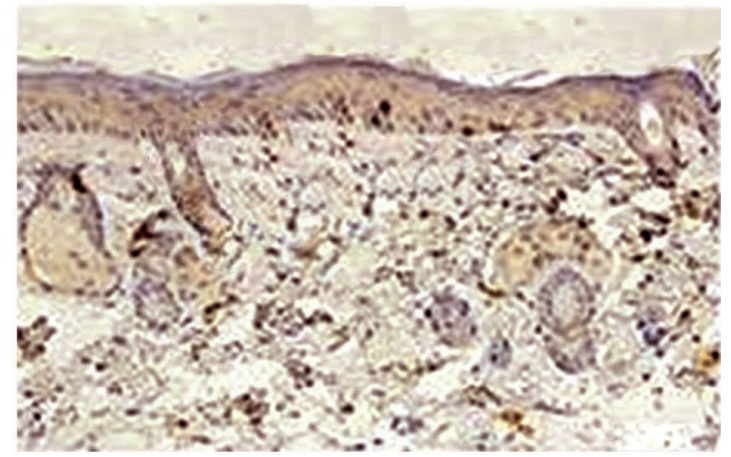

Figure 2. Oleuropein treatment suppressed infiltration of T cell-mediated inflammatory cells. A - CD3+ cells in IMQ-mediated psoriasis mice

${ }^{*} P<0.05$ and ${ }^{* *} p<0.01$ compared to IMQ-induced mice. 


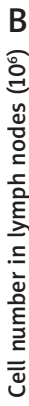

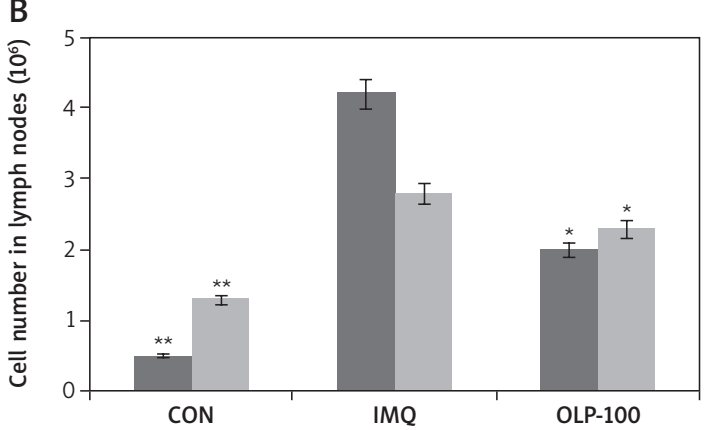

CD4 $\quad$ CD8
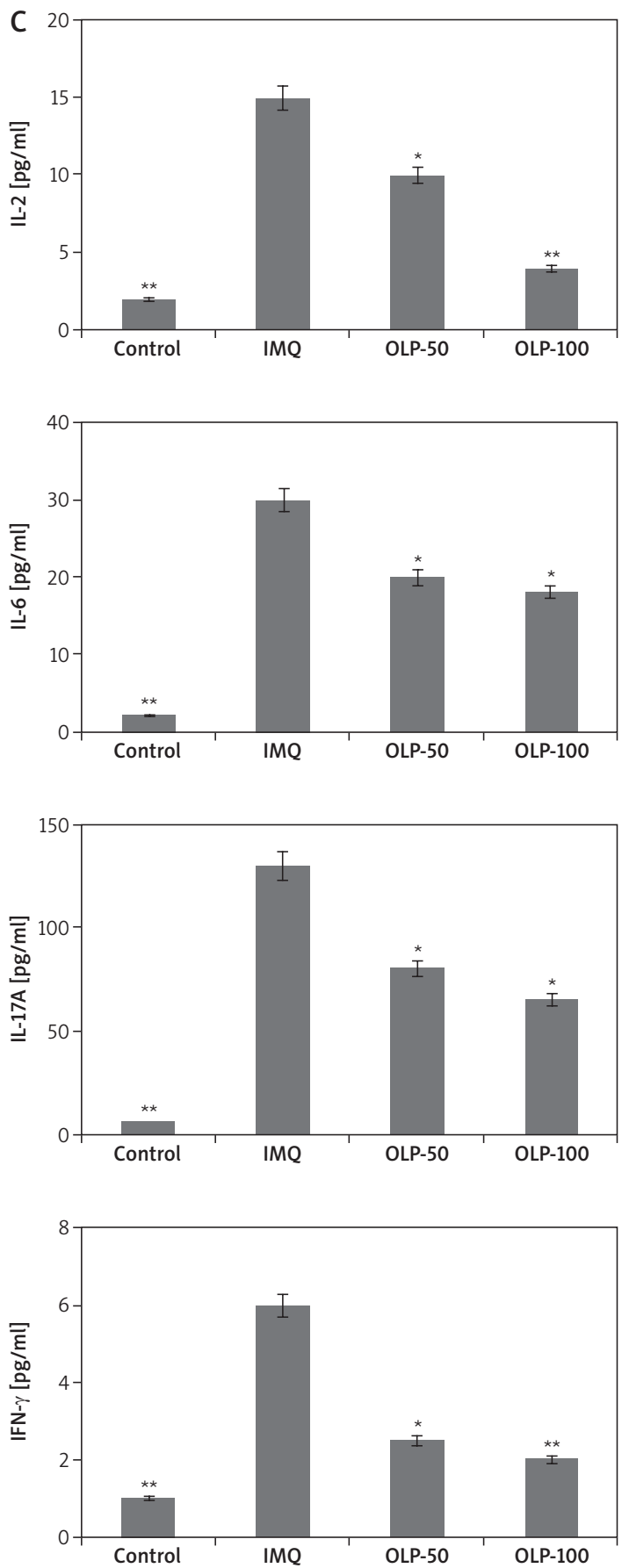

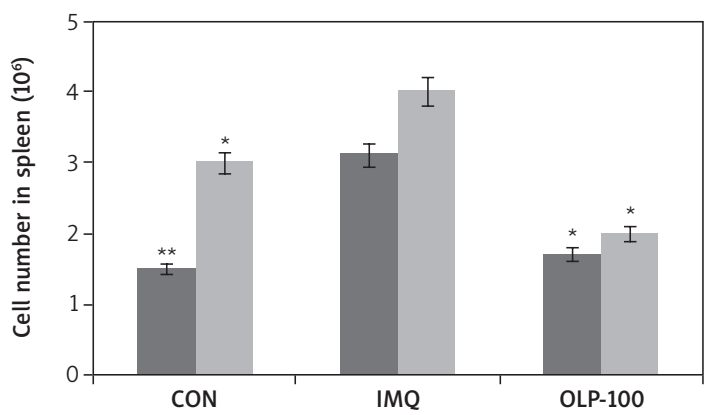

CD4 CD8
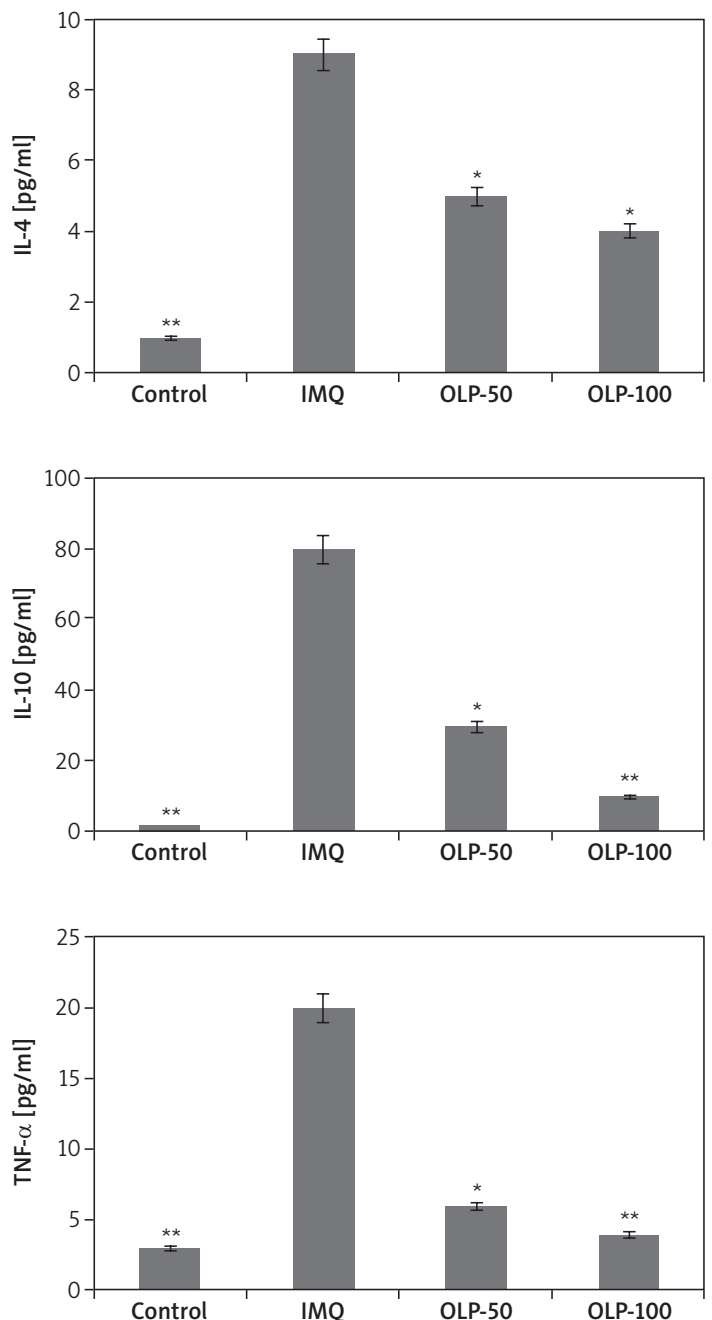

Figure 2. Cont. B - Number of CD4+ and CD8+ cells in spleen and lymph nodes. C - Levels of inflammatory cytokines related to psoriasis by beadbased immunoassay

${ }^{*} P<0.05$ and ${ }^{* *} P<0.01$ compared to IMQ-induced mice. 


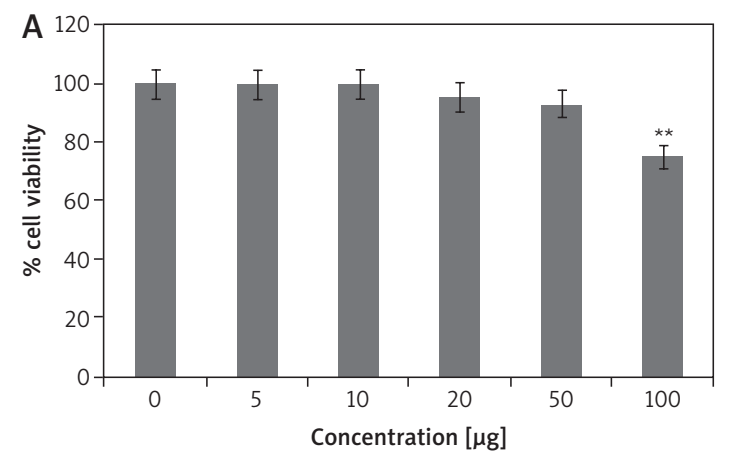

Figure 3. Oleuropein treatment suppressed the functioning and differentiation of Th17 cells. A - Results of CCK-8 assay for cell viability of OLPtreated CD4+ T cells. B - Results of flow cytometry for analysis of IL-17A levels in OLP-treated CD4+ T cells

${ }^{*} P<0.05$ and ${ }^{* *} p<0.01$ compared to $C D 4+T$ cells devoid of OLP or IMP treated mice.
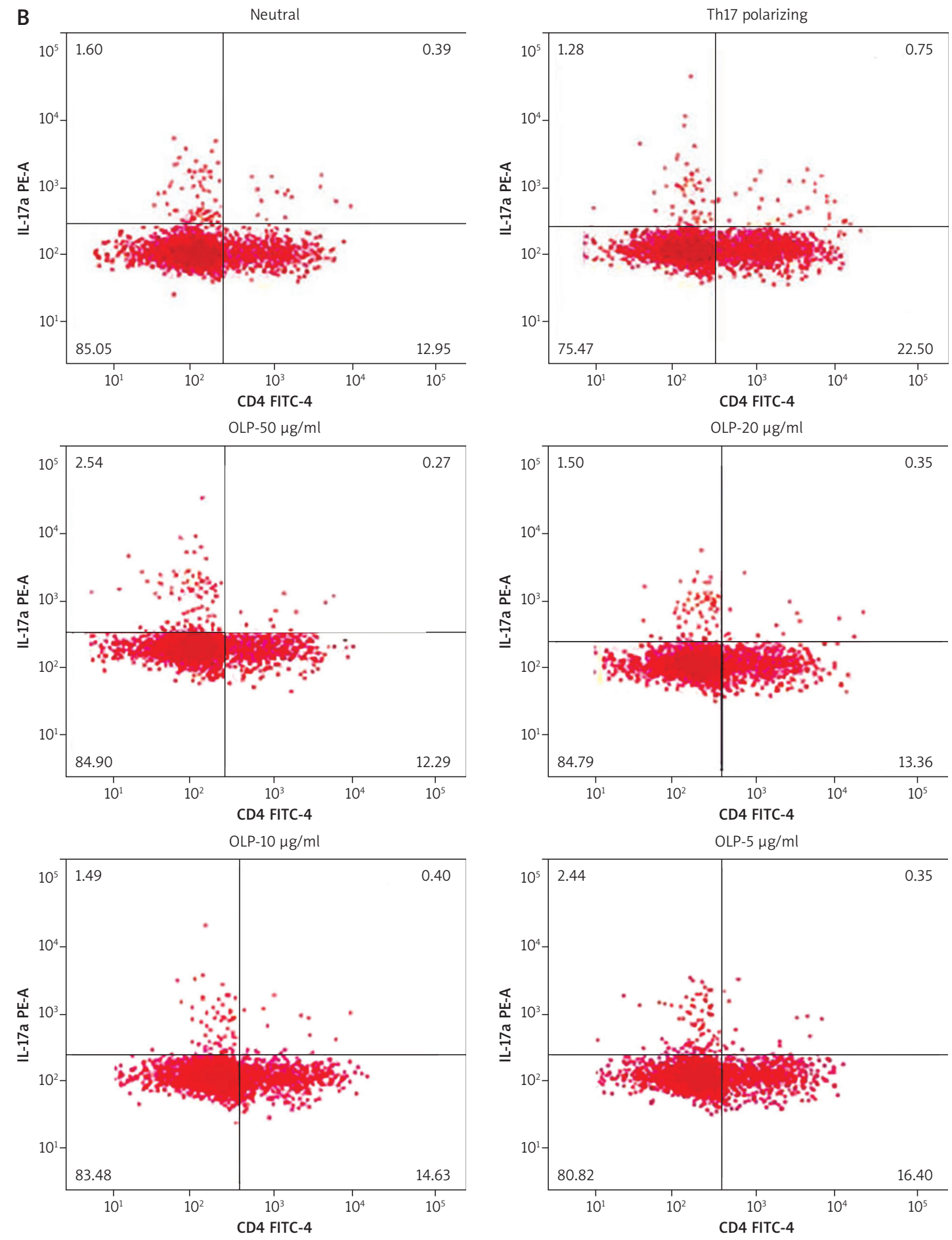

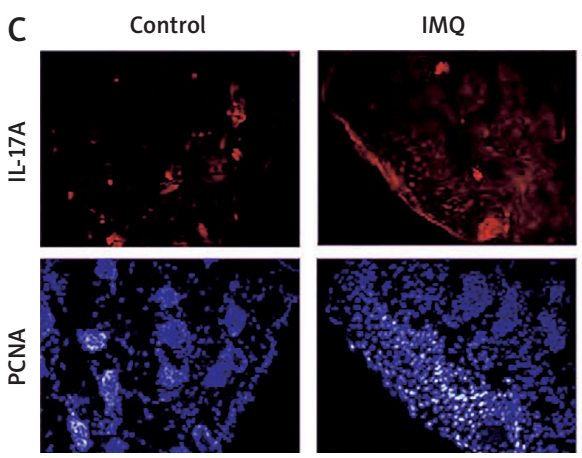

MTX
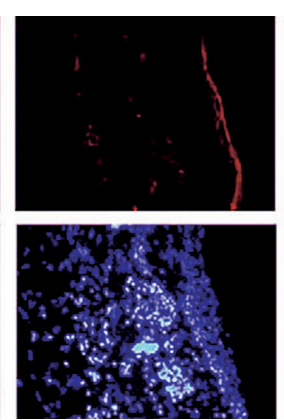

OLP-50
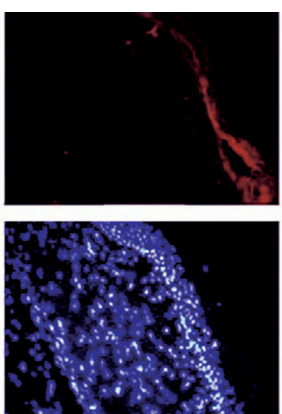

OLP-100

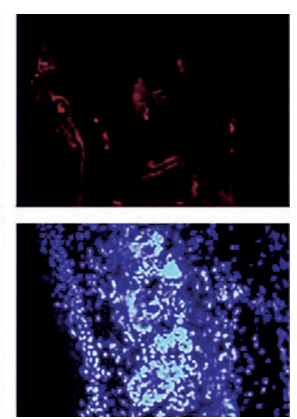

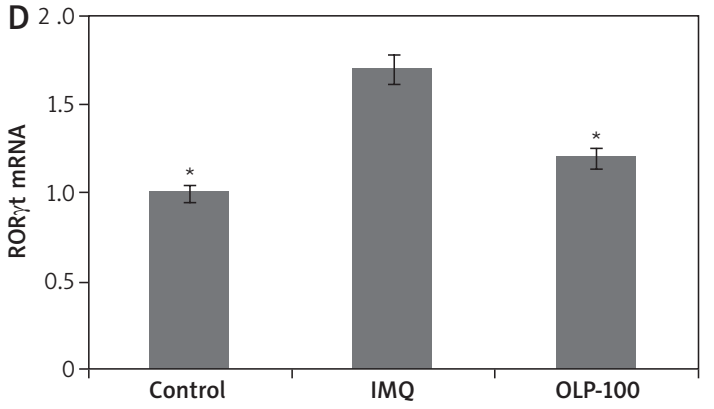

Figure 3. Cont. Oleuropein treatment suppressed the functioning and differentiation of Th17 cells. C - Immunofluorescence images of IL-17A positive cells in IMQ mice treated with MTX, OLP (50 and $100 \mathrm{mg} / \mathrm{kg}$ ). Red color = IL-17A and blue color = DAPI positive. $\mathrm{D}-\mathrm{mRNA}$ expression of ROR $\gamma \mathrm{t}$ in skin lesions of IMQ-induced mice

${ }^{*} P<0.05$ and ${ }^{* *} p<0.01$ compared to CD4+ $T$ cells devoid of OLP or IMP treated mice. and after incubating with OLP. OLP suppressed the Th17 differentiation and also the release of IL$17 \mathrm{~A}$ with increasing dose in the supernatants (Figure 3 B). In the PSR mouse model, IL-17A was found in both dermal layer and epidermal keratinocytes, but in OLP mice decreased IL-17A+ cell infiltration was observed in the skin lesions (Figure $3 \mathrm{C}$ ) along with a decrease in levels of mRNA Th17 specific transcription factor ROR $\gamma$ t (Figures 3 C, D). These findings indicated that OLP inhibits the functioning and differentiation of cells.

\section{Oleuropein treatment inhibitory effect} is propagated via the JAK3/STAT3 pathway in psoriatic mice

Previously, STAT3 has been found to regulate differentiation of Th17 cells and keratinocytes $[23,24]$. To study whether OLP alters Th17 cells via STAT3 in the skin lesions of PSR mice, we performed western blot analysis and found over-expression of PSTAT3 and pJAK3. Treatment with OLP at high dose $(100 \mu \mathrm{g} / \mathrm{ml})$ in PSR mice suppressed the levels of both STAT3/JAK and pSTAT3/pJAK. In addition, OLP treatment elevated the expression of SCOSE3 in PSR mice (Figure 4). These outcomes suggested that OLP might block the cell activity of Th17 cells by targeting STAT3/JAK proteins.

\section{Oleuropein had higher binding affinity with STAT3 and JAK3}

In view of the in vivo and in vitro outcomes, a molecular docking study was performed by BLAST and homology modeling followed by ligand and protein preparation, the molecular docking simulation of the ligand OLP was done by Autodock Kollman and Gasteiger charges in both the ligand and protein. The docking results showed higher binding affinity for ligand OLP with both the in vivo confirmed proteins STAT3 and JAK3. The binding energies showed that ligand OLP had lower binding energies with STAT3 (Table I). The 3D structure of protein showed solid area representing the binding area of ligand in protein in both STAT3 and JAK3 (Figure 5).

\section{Discussion}

The present work evaluated the impression of OLP in PSR mice. We developed an IMQmediated PSR model in BALB/C mice showing skin lesions and evidenced that treatment of OLP ameliorated the proliferation of keratinocytes and inflammatory infiltration significantly; the treatment also improved the cellular, molecular and histopathological features. OLP decreased the CD3+ cell infiltration in the lesions and increased circulating cytokines and CD4 and CD8+ T cells. OLP at a dose of $100 \mathrm{mg} / \mathrm{kg}$ showed comparably highly improved results compared to the lower dose of $50 \mathrm{mg} / \mathrm{kg}$ in mice; still, more studies are needed to confirm the dose. In vitro results suggested that OLP inhibited Th17 cell differentiation, regulated levels of IL-17A along with JAK/STAT3 in Th17 cells. OLP also resulted in over-expression of SCOSE3 in PSR mice. The results hence indicate that OLP could be a potential molecule to antagonize the local inflammation by mediating the function of Th17 cells in 


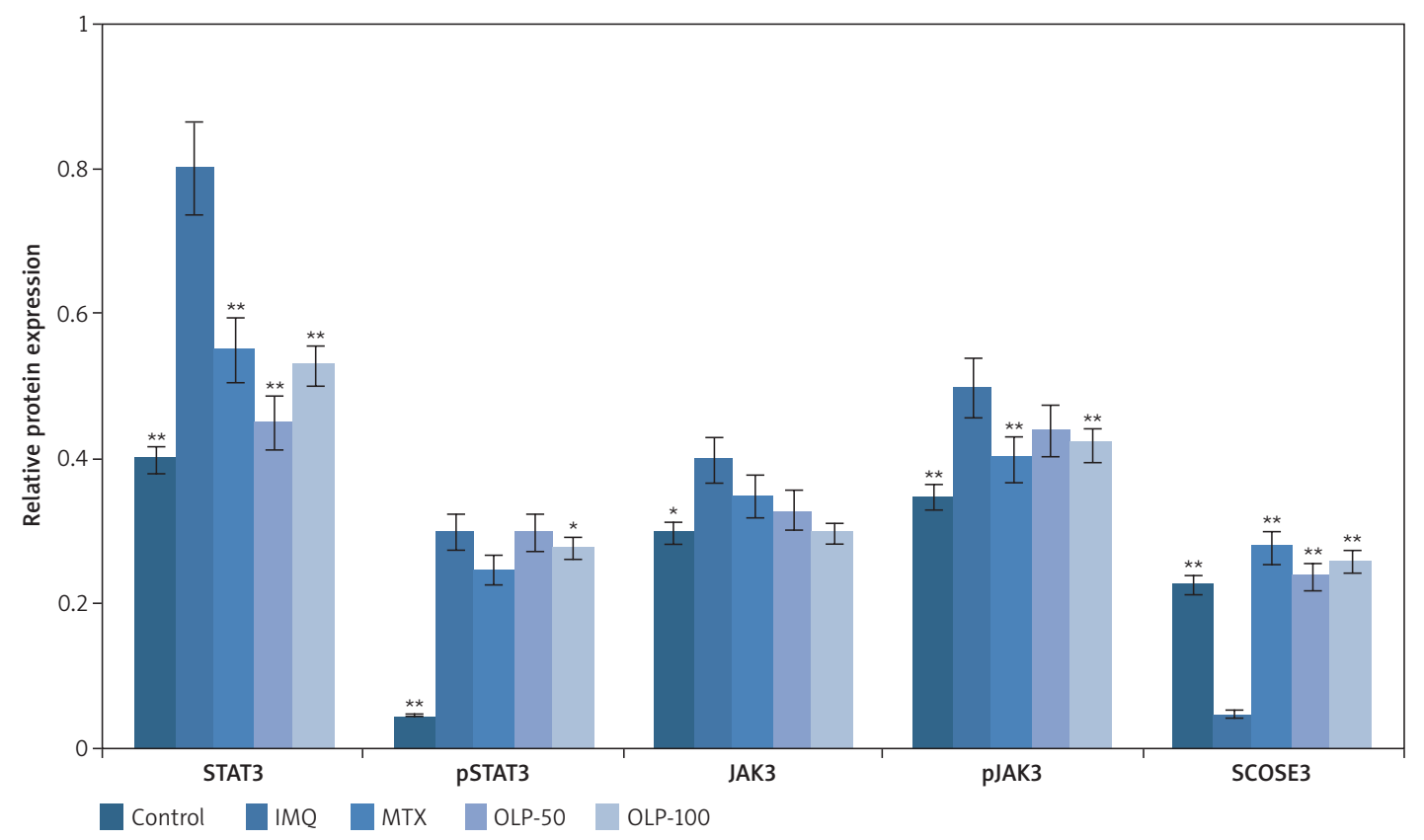

Figure 4. Oleuropein treatment suppressed activation of STAT3 cascade. Quantitative expression levels of STAT3 and pSTAT3, JAK3 and pJAK3 showed treatment of OLP decreased the levels. The expression of SCOSE3 was increased in mice treated with OLP compared to IMQ control mice

${ }^{*} P<0.05$ and ${ }^{* *} P<0.01$ compared to IMQ-treated mice.

Table I. Docking scores for protein STAT3 and JAK3 with the ligand molecule OLP

\begin{tabular}{|c|c|c|c|}
\hline $\begin{array}{l}\text { Mode } \\
\text { of ligand }\end{array}$ & $\begin{array}{c}\text { Affinity } \\
{[\mathrm{kcal} / \mathrm{mol}]}\end{array}$ & $\begin{array}{c}\text { Distance } \\
\text { from rmsd l.b }\end{array}$ & $\begin{array}{c}\text { Best mode } \\
\text { rmsd u.b }\end{array}$ \\
\hline \multicolumn{4}{|c|}{ Docking score for STAT3 protein and OLP: } \\
\hline 1 & -5.4 & 0.00 & 0.00 \\
\hline 2 & -5.4 & 12.850 & 17.646 \\
\hline 3 & -5.2 & 11.795 & 15.831 \\
\hline 4 & -5.5 & 2.236 & 7.721 \\
\hline 5 & -5.1 & 14.652 & 18.338 \\
\hline 6 & -5.0 & 13.982 & 17.211 \\
\hline 7 & -5.0 & 12.418 & 15.249 \\
\hline 8 & -4.9 & 12.804 & 16.830 \\
\hline 9 & -4.8 & 13.703 & 18.005 \\
\hline \multicolumn{4}{|c|}{ Docking score for JAK3 protein and OLP: } \\
\hline 1 & -7.6 & 0.000 & 0.000 \\
\hline 2 & -7.3 & 3.015 & 7.908 \\
\hline 3 & -7.0 & 3.053 & 7.318 \\
\hline 4 & -7.0 & 2.676 & 7.103 \\
\hline 5 & -6.8 & 4.758 & 7.690 \\
\hline 6 & -6.7 & 16.382 & 19.971 \\
\hline 7 & -6.7 & 2.826 & 5.981 \\
\hline 8 & -6.7 & 16.862 & 19.824 \\
\hline 9 & -6.6 & 16.611 & 19.975 \\
\hline
\end{tabular}

PSR mice. Previous studies have reported that OLP suppresses the activity of T cells [25]. In line with this, we evidenced that OLP decreased the T cell count in the PSR skin lesions and decreased the CD8+T and CD4+ count in both lymph nodes and spleen. The variation in count of $C D 8+T$ cells was less significant compared to the relative number of cells (\%).

Imiquimod (IMQ) is a toll like receptor and immune activator which on topical application aggravates inflammatory skin lesions; it is used in studies for creating an animal model of PSR [20]. The model is a replica of the human condition of PSR which includes hyperkeratosis, erythema, scaling and neutrophil and T cell infiltration in skin. Th17 cells are reported to be the dominant cells in pathogenesis of plaque PSR [26]. In our study, $T$ cells and dendritic cells were found in spleen of mice treated with IMQ, in addition which, CD4+ IL17+ T cells were found to be upregulated, although no changes were observed in the number of CD4+ IFN- $\gamma+$ T cells. Treatment with OLP ameliorated the blood levels of IL-17A as well as in skin lesions. OLP suppressed the percentage of CD4IL$17 \mathrm{~A}+$ cells and also the expression of IL-17A, indicating that OLP suppresses the differentiation of Th17 cells along with functioning of Th17 cells, leading to suppression of Th17-linked inflammation. It is reported that CD8+ cells produce IL-17. In our study the number of CD8+ cells was altered in lymph nodes as well as in spleen of OLP-treated IMQ mice; hence more studies are required which could evaluate the role of OLP in T cell IL-17 levels. 

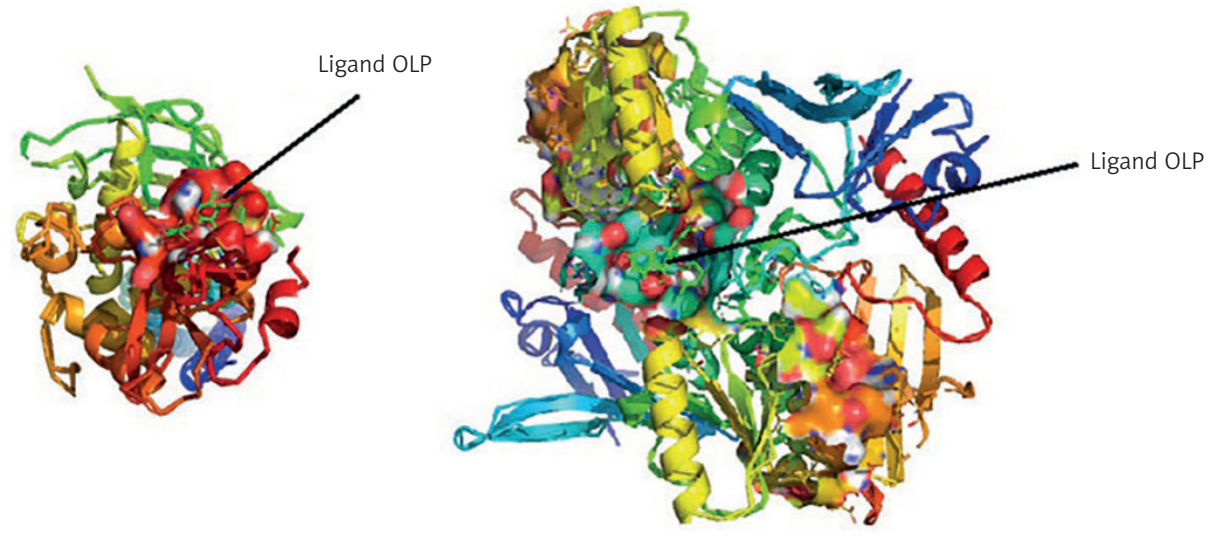

Protein STAT3 with ligand OLP

Protein JAK3 with ligand OLP

Figure 5. In silico molecular docking showed interaction of protein STAT3 and JAK3 with ligand oleuropein. The solid area in protein structures represents the area of interaction with ligand oleuropein

In the mouse of model of PSR, OLP suppressed the expression of TNF- $\alpha$ and also that of Th17related cytokine IFN- $\gamma$. The OLP treatment also attenuated the IMG-mediated levels of IL-10. We found that OLP inhibits IL-17, which also leads to decreased expression of IL-10, but these findings may be specific to the IMQ-induced PSR model as we do not find any reports describing the same observations. The effect of OLP is in agreement with an earlier report in which OLP extended the inhibitory effect on secretion of cytokines including IL-10 [27]. A number of studies have demonstrated that STAT3 is over-expressed in PSR and also in an IMQ-induced PSR model [28]. JAK3 is found to be involved in activation of $T$ cells and their proliferation $[29,30]$. SCOSE3 is reported to regulate STAT3 negatively and ROR $\gamma \mathrm{t}$, which is a downstream effector of STAT3 in the activation of Th17 cells. We also found that OLP suppressed the levels of pSTAT3 and pJAK3 and also decreased the levels of RORyt, suggesting that OLP may inhibit Th17 cells involving the JAK3/STAT3 cascade. Also, OLP treatment over-expressed the levels of SCOCE3, indicating that OLP targets multiple processes associated with STAT3 signaling in a PSR mouse model. However, more research is required for determining the specific molecular cascade involved in treatment with OLP.

In light of the in vivo and in vitro results, a molecular docking study was performed to further confirm the ligand and protein interaction scores using in silico bioinformatics tools. The molecular docking studies suggested that OLP bound with both STAT3 and JAK3 protein with minimal energy and high binding affinity, thus further strengthening the in vivo and in vitro findings.

In conclusion, the present study demonstrates that OLP inhibits IMQ-mediated PSR-like inflammation by decreasing differentiation of inflam- matory cells and levels of cytokines, specifically suppressing the Th17 differentiation and levels of IL-17A. Outcomes suggested a new therapeutic molecule for treating this inflammatory skin disease, and suggest that OLP could be a potential molecule for inhibiting PSR. However, studies of the present molecule involving other PSR models are still needed.

\section{Acknowledgments}

The authors are thankful to Hubei Provincial Hospital of Traditional Chinese Medicine China. The authors are also grateful to the funding agencies basic scientific research expenses of public welfare research institutes at the central level selected topics independently by the Foundation Institute of The China Academy of Chinese Medical Sciences for providing necessary funds for experiments.

This study was supported by basic scientific research expenses of public welfare research institutes at the central level selected topics independently by the Foundation Institute of The China Academy of Chinese Medical Sciences (No. YZ-1743).

\section{Conflict of interest}

The authors declare no conflict of interest.

\section{References}

1. Nestle FO, Kaplan DH, Barker J. Psoriasis. N Engl J Med 2009; 361: 496-509.

2. Becher B, Pantelyushin S. Hiding under the skin: interleukin-17-producing gammadelta $T$ cells go under the skin? Nat Med 2012; 18: 1748-50.

3. Gutcher I, Becher B. APC-derived cytokines and T cell polarization in autoimmune inflammation. J Clin Invest 2007; 117: 1119-27.

4. Martin DA, Towne JE, Kricorian G, Klekotka P, Gudjonsson JE, Krueger JG. The emerging role of IL-17 in the 
pathogenesis of psoriasis: preclinical and clinical findings. J Investig Dermatol 2013; 133: 17-26.

5. Afzali B, Lombardi G, Lechler RI, Lord GM. The role of T helper 17 (Th17) and regulatory T cells (Treg) in human organ transplantation and autoimmune disease. Clin Exp Immunol 2007; 148: 32-46.

6. Lowes MA, Suarez-Farinas M, Krueger JG. Immunology of psoriasis. Annu Rev Immunol 2014; 32: 227-55.

7. Lynde CW, Poulin Y, Vender R, Bourcier M, Khalil S. Interleukin 17A: toward a new understanding of psoriasis pathogenesis. J Am Acad Dermatol 2014; 71: 141-50.

8. Langley RGB, Krueger GG, Griffiths CEM. Psoriasis: epidemiology, clinical features, and quality of life Ann Rheum Dis 2005; 64: 18-23.

9. Chandrakumar SF, Yeung J. Interleukin-17 antagonists in the treatment of psoriasis. J Cutan Med Surg 2015; 19. 109-14.

10. Al-Waili NS. Topical application of natural honey, beeswax and olive oil mixture for atopic dermatitis or psoriasis: partially controlled, single-blinded study. Complementary Therapies in Medicine 2003; 11: 226-34.

11. Santangelo C, Vari R, Scazzocchio B, et al. Anti-inflammatory activity of extra virgin olive oil polyphenols: which role in the prevention and treatment of immunemediated inflammatory diseases? Endocr Metab Immune Disord Drug Targets 2018; 18: 36-50.

12. Gambino CM, Accardi G, Aiello A, Candore et al. Effect of extra virgin olive oil and table olives on the immune inflammatory responses: potential clinical applications. Endocr Metab Immune Disord Drug Targets 2018; 18: 14-22.

13. Wainstein J, Ganz T, Boaz M, et al. Olive leaf extract as a hypoglycemic agent in both human diabetic subjects and in rats. J Med Food 2012; 15: 605-10.

14. Boss A, Bishop KS, Marlow G, Barnett MP, Ferguson LR. Evidence to support the anti-cancer effect of olive leaf extract and future directions. Nutrients 2016; 8: 513.

15. Magrone T, Spagnoletta A, Salvatore R, et al. Olive leaf extracts act as modulators of the human immune response. Endocr Metab Immune Disord Drug Targets 2018; 18: 85-93.

16. Giner E, Recio MC, Ríos JL, Cerda-Nicolas JM, Giner RM. Chemopreventive effect of oleuropein in colitis-associated colorectal cancer in c57bl/6 mice. Mol Nutr Food Res 2016; 60: 242-55.

17. Khalatbary AR, Zarrinjoei GR. Anti-inflammatory effect of oleuropein in experimental rat spinal cord trauma. Iran Red Crescent Med J 2012; 14: 229-34.

18. Qin S, Wen J, Bai XC, Chen TY, Zheng RC, Zhou GB. Endogenous $n-3$ polyunsaturated fatty acids protect against imiquimod-induced psoriasis-like inflammation via the IL-17/IL-23 axis. Mol Med Rep 2014; 9: 2097-104.

19. Baharvand B, Esmailidehaj M, Alihosaini J, Bajoovand S, Esmailidehaj S, Hafizie Z. Prophylactic and therapeutic effects of oleuropein on reperfusion-induced arrhythmia in anesthetized rat. Iran Biomed J 2016; 20: 41-8.

20. Van der Fits L, Mourits S, Voerman JS, Kant M, Boon L, Laman JD. Imiquimod-induced psoriasis-like skin inflammation in mice is mediated via the IL-23/IL-17 axis. J Immunol 2009; 182: 5836-45.

21. Zhou Z, Sun W, Liang Y, Gao Y, Kong W, Guan Y. Fenofibrate inhibited the differentiation of $T$ helper 17 cells in vitro. PPAR Res 2012; 2012: 145654.

22. Xu Y, Li Z, Yin Y, Lan H, Wang J, Zhao J. Ghrelin inhibits the differentiation of $T$ helper 17 cells through mTOR/ STAT3 signaling pathway. PLoS One 2015; 10 : e0117081.
23. Rebe C, Vegran F, Berger H, Ghiringhelli F. STAT3 activation: a key factor in tumor immunoescape. JAKSTAT 2013; 2: e23010.

24. Simanski M, Rademacher F, Schroder L, Schumacher HM, Glaser R, Harder J. IL-17A and IFN-gamma synergistically induce RNase 7 expression via STAT3 in primary keratinocytes. PLoS One 2013; 8: e59531.

25. Larussa T, Oliverio M, Suraci E, et al. Oleuropein decreases cyclooxygenase-2 and interleukin-17 expression and attenuates inflammatory damage in colonic samples from ulcerative colitis patients. Nutrients 2017; 9: 391.

26. Vajdian R, Mosaffa N, Tabaei SJS, et al. Effect of olive leaf extract on cytokines secreted by macrophage. Novelty in Biomedicine 2016; 4: 116-20.

27. Gaffen SL, Jain R, Garg AV, Cua DJ. The IL-23-IL-17 immune axis: from mechanisms to therapeutic testing. Nat Rev Immunol 2014; 14: 585-600.

28. Lu X, Du J, Liang J, Zhu X, Yang Y, Xu J. Transcriptional regulatory network for psoriasis. J Dermatol 2013; 40: 48-53.

29. Andres RM, Hald A, Johansen C, Kragballe K, Iversen L. Studies of Jak/STAT3 expression and signalling in psoriasis identifies STAT3-Ser727 phosphorylation as a modulator of transcriptional activity. Exp Dermatol 2013; 22 323-8.

30. Mysliwiec H, Harasim-Symbor E, Baran A, et al. Abnormal serum fatty acid profile in psoriatic arthritis. Arch Med Sci 2019; 15: 1407-14. 